\title{
Mixed convection flow of nanofluid with Hall and ion-slip effects using spectral relaxation method
}

\author{
Wubshet Ibrahim ${ }^{1^{*}}$ (I) and Temesgen Anbessa ${ }^{2}$
}

\author{
* Correspondence: wubshetib@ \\ yahoo.com \\ ${ }^{1}$ Department of Mathematics, Ambo \\ University, Ambo, Ethiopia \\ Full list of author information is \\ available at the end of the article
}

\begin{abstract}
In this article, the Hall and ion-slip effects on a mixed convection flow of an electrically conducting nanofluid over a stretching sheet in a permeable medium have been discussed. Using the similarity transformations, the partial differential equations corresponding to the momentum, energy, and concentration equations are transformed to a system of nonlinear ordinary differential equations which are solved numerically using a spectral relaxation method (SRM). The effects of significant parameters on the velocities, temperature, and concentration profiles are analyzed graphically. Moreover, the results of the skin friction coefficients, local Nusselt number, and Sherwood number are determined numerically. The results of the analysis showed that the velocity profile in the flow direction increases with an increase in mixed convection parameter $\lambda$, Hall parameter $\beta_{h}$, and ion-slip parameter $\beta_{i}$, and it decreases with an increase in the magnetic parameter M. Furthermore, temperature and concentration profiles decrease as the mixed convection parameter $\lambda$ and buoyancy ratio $\mathrm{Nr}$ increase. It is also observed that the skin friction coefficients, local Nusselt number, and Sherwood number increase with an increase in the Hall parameter $\beta_{h}$, mixed convection parameter $\lambda$, and buoyancy ratio $N$ r.
\end{abstract}

Keywords: Mixed convection, Nanofluid, Hall and ion-slip effects, SRM

\section{Introduction}

Recently, the study of convection and heat transfer in the presence of nanofluid has obtained significant attention because of its wide applications in science, engineering, and industry. Some of these applications are welding equipment, power generating systems, cooling of nuclear reactor, automobile engines, and heat exchanging in electronics devices. The concept of nanofluid was first commenced by Choi [1] to refer to the fluids with suspended nanoparticles $(1-100 \mathrm{~nm})$. The suspended nanoparticles assist to enhance the thermal conductivity of the fluid. The effects of various governing parameters on heat and mass transfer of nanofluid flow through different geometries were studied by different scholars ([2-10]). Li et al. [11] numerically modeled electrohydrodynamic nanoparticle flow using control volume-based finite element method. Ramzan et al. [12] analyzed the aqueous-based nanofluid flow containing carbon nanotubes past a vertical cone through a porous medium with entropy generation and thermal radiation. Bilal and Ramzan [13] discussed unsteady two-dimensional flow of mixed

(c) The Author(s). 2019 Open Access This article is distributed under the terms of the Creative Commons Attribution 4.0 International License (http://creativecommons.org/licenses/by/4.0/), which permits unrestricted use, distribution, and reproduction in any medium, provided you give appropriate credit to the original author(s) and the source, provide a link to the Creative Commons license, and indicate if changes were made. 
convection and nonlinear thermal radiation in the presence of water-based carbon nanotubes over the vertically convected stretched sheet embedded in a DarcyForchheimer porous media using Saffman's proposed model for the suspension of fine dust particles in the nanofluid. Lu et al. [14] scrutinized a thin film flow of a nanofluid consisting of single and multi-walled carbon nanotubes with Cattaneo-Christov heat flux and entropy generation. Suleman et al. [15] studied the impacts of Newtonian heating and homogeneous-heterogeneous reactions on the flow of silver-water nanofluid past a nonlinear stretched cylinder with MHD and nonlinear thermal radiation. Suleman et al. [16] extended the work of Suleman et al. [15] by incorporating viscous dissipation, heat generation/absorption and joule heating effects. Madhu et al. [17] studied the boundary layer flow and heat transfer of a power-law non-Newtonian nanofluid past a nonlinearly stretching sheet. Madhu and Kishan [18] numerically investigated MHD mixed convection flow of heat and mass transfer stagnation-point flow of a non-Newtonian power-law nanofluid towards a stretching surface in the presence of thermal radiation using finite element method. Macha et al. [19] analyzed the MHD boundary layer flow of a viscous, incompressible and electrically conducting nonNewtonian nanofluid obeying power-law model over a nonlinear stretching sheet under the effect of thermal radiation with heat source/sink. Alebraheem and Ramzan [20] studied the heat and mass transfer of Casson nanofluid flow containing gyrotactic microorganisms past a swirling cylinder. Farooq et al. [21] described the static wedge flow of non-viscous Maxwell nanofluid using BVPh2.0 solver. Farooq et al. [22] analyzed the effects of thermophoretic and Brownian motion on MHD non-Newtonian Maxwell fluid with nanomaterials over an exponentially stretching surface using Buongiorno model. Macha et al. [23] investigated the MHD boundary layer flow of Sisko nanofluid flow past a wedge.

Mixed convection flows got a significant consideration because of its numerous technological and industrial applications. Aman and Ishak [24] numerically considered the effects of buoyancy force and convective surface boundary condition on mixed convection flow near a resistant vertical plate using shooting technique. Mahanthesh et al. [25] numerically investigated the influences of buoyancy force, nanoparticles, chemical reaction, and heat source/sink on combined forced and free convection flow of an electrically conducting water-based $\mathrm{Cu}$-nanofluid past a moving/stationary vertical plate using Laplace transform method. The impacts of different governing parameters on mixed convection flow of nanofluid past stretching surfaces were studied by ([26-28]). Most recently, Khan and Rasheed [29] scrutinized magnetohydrodynamic (MHD) combined natural and forced convection flow of the Maxwell nanofluid with magnetic field and buoyancy force effects.

The Hall and ion-slip impacts are important under the existence of strong magnetic field because of the significant effect they have on the magnitude and direction of the electric current density and the magnetic force term. Hence, in numerous physical circumstances, it is necessary to incorporate the impact of Hall current and ion-slip terms in the MHD equations. The impacts of Hall and ion-slip parameters on combined forced and free convection flow of an electrically conducting Casson fluid were numerically analyzed using homotopy analysis method by Reddy et al. [30]. Moreover, Reddy et al. [31] analyzed the effects of Hall and ion-slip effects on combined forced and free convection flow of an electrically conducting Newtonian fluid using Adomian decomposition method. The Hall and ion-slip effects on mixed convection flow of an 
electrically conducting nanofluid through different geometries were discussed by ([3235]). Motsa and Shateyi [36] numerically analyzed the effects of Hall and ion-slip currents on magnetomicropolar fluid flow with suction/injection using the spectral linearization method. Moreover, using shooting method, Bilal et al. [37] extended the work of Motsa and Shateyi [36] with the inclusion of nanoparticles neglecting the effect of chemical reaction. The Hall and ion-slip effects on unsteady MHD Couette flow of an electrically conducting fluid has been analytically discussed using Laplace transform method by Ghara et al. [38].

Ali et al. [39] have studied the effect of Hall current on MHD mixed convection flow over a stretched vertical flat plate. However, they did not consider ion-slip and permeability effects. Thus, the objective of the present study is to investigate the effects of Hall and ion-slip parameter on mixed convection flow of nanofluid past a porous stretching sheet. Therefore, the inclusion of the effects of ion-slip and permeability parameters in the presence of mass transfer makes this study a novel one. The governing equations are solved numerically using the SRM. This numerical method is chosen since its accuracy is high and the MATLAB program for the problem is manageable (see: Motsa and Makukula [40], Shateyi and Marewo [41], Shateyi and Marewo [42]). The fluid model considered in this study is solved numerically and hence will present an approximated solution. The other limitation of this flow problem is that the nanoparticle volume fraction is neglected.

\section{Mathematical formulation}

The study considered a laminar mixed convection flow of a viscous, incompressible, and electrically conducting nanofluid past a vertical linearly stretching sheet through a permeable medium with velocity $V_{w}(x)$ in the direction of $x$-axis. A strong uniform magnetic field $M_{0}$ is applied in the way along $y$-axis as shown in Fig. 1. As a result of this strong magnetic field force, the electrically conducting fluid has the Hall and ionslip effects which generate a cross-flow in the $z$-direction, and thus, the flow becomes three dimensional. The induced magnetic field can be ignored in contrast with the applied magnetic field on the presumption that the flow is steady and the magnetic Reynolds number is very small. The mixed convection flow is caused by the buoyancy

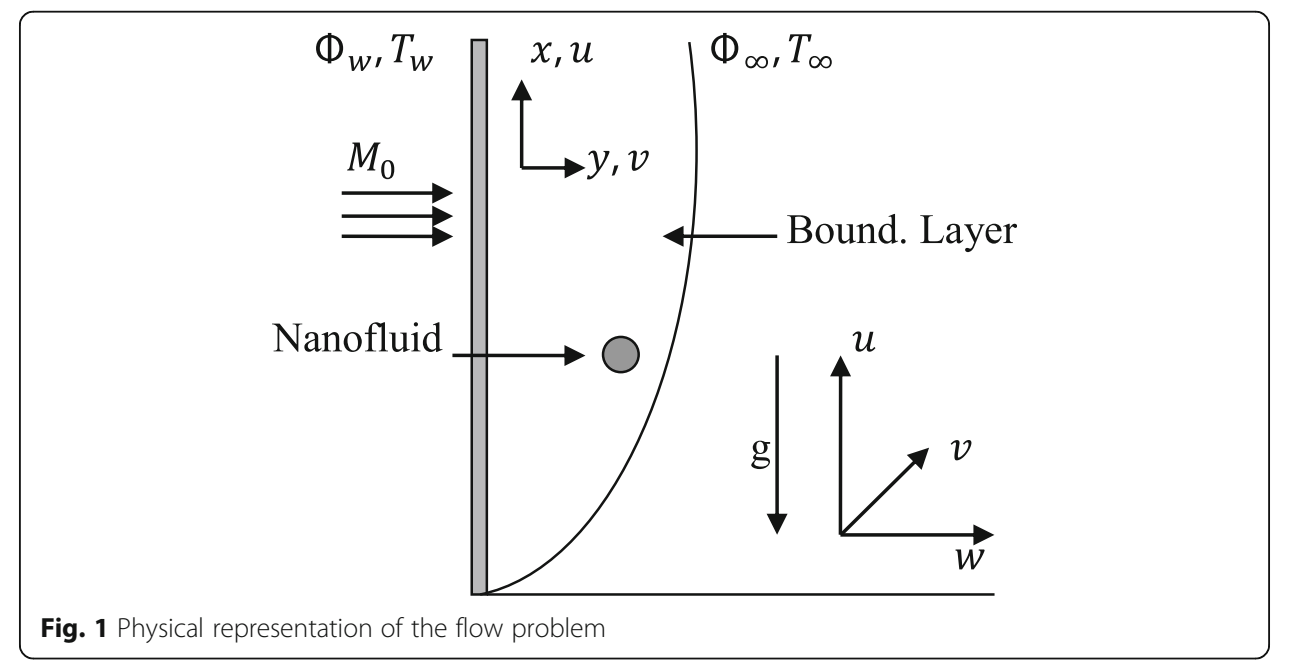


forces. Further, all the fluid properties are understood to be constant except for density variations in the buoyancy force term. By applying the Boussinesq approximations with the above assumptions, the governing equations of continuity, momentum, energy, and concentration equations for the considered flow problem are $([35,37,38])$ :

$$
\begin{aligned}
& \frac{\partial u}{\partial x}+\frac{\partial v}{\partial y}=0 \\
& u \frac{\partial u}{\partial x}+v \frac{\partial u}{\partial y}=v \frac{\partial^{2} u}{\partial y^{2}}-\frac{\sigma M_{0}^{2}}{\rho\left(\beta^{2}+\beta_{h}^{2}\right)}\left(\beta u+\beta_{h} w\right) \\
& +\mathrm{g}\left[\mathrm{B}_{\mathrm{T}}\left(\mathrm{T}-\mathrm{T}_{\infty}\right)+\mathrm{B}_{\mathrm{C}}\left(\Phi-\Phi_{\infty}\right)\right]-\frac{v}{\kappa} u \\
& u \frac{\partial w}{\partial x}+v \frac{\partial w}{\partial y}=v \frac{\partial^{2} w}{\partial y^{2}}+\frac{\sigma M_{0}^{2}}{\rho\left(\beta^{2}+\beta_{h}^{2}\right)}\left(\beta_{h} u-\beta w\right)-\frac{v}{\kappa} w \\
& u \frac{\partial T}{\partial x}+v \frac{\partial T}{\partial y}=\alpha \frac{\partial^{2} T}{\partial y^{2}}+\gamma\left[D_{B} \frac{\partial T}{\partial y} \frac{\partial \Phi}{\partial y}+\frac{D_{T}}{T_{\infty}}\left(\frac{\partial T}{\partial y}\right)^{2}\right] \\
& u \frac{\partial \Phi}{\partial x}+v \frac{\partial \Phi}{\partial y}=D_{B} \frac{\partial^{2} \Phi}{\partial y^{2}}+\frac{D_{T}}{T_{\infty}} \frac{\partial^{2} T}{\partial y^{2}}
\end{aligned}
$$

where $(u, v, w)$ are the fluid velocity components along the $(x, y, z)$ coordinates, $\mu$ is the dynamic viscosity, $\rho$ is the density, $v$ is the kinematic viscosity, $g$ is the acceleration due to gravity, $T$ and $\Phi$ are the fluid temperature and concentration, respectively, $T_{\infty}$ and $\phi_{\infty}$ are the ambient temperature and concentration, respectively, $B_{T}$ is the coefficient of thermal expansion, $B_{C}$ is the solutal coefficient of expansion, $\sigma$ is the electrical conductivity, $M_{0}$ is the constant magnetic field applied normal to the surface, $\beta_{h}$ is the Hall parameter, $\beta_{i}$ is the ion-slip parameter, $\beta=1+\beta_{h} \beta_{i}$ is a constant, $\kappa$ is the permeability of the porous medium, $\alpha$ is the thermal diffusivity, $(\rho \Phi)_{p}$ is the heat capacity of the nanoparticle, $\gamma$ is the ratio of the effective heat capacity of nanoparticle material to the heat capacity of the fluid, $D_{B}$ the Brownian diffusion coefficient, and $D_{T}$ the thermophoresis diffusion coefficient.

The corresponding boundary conditions are:

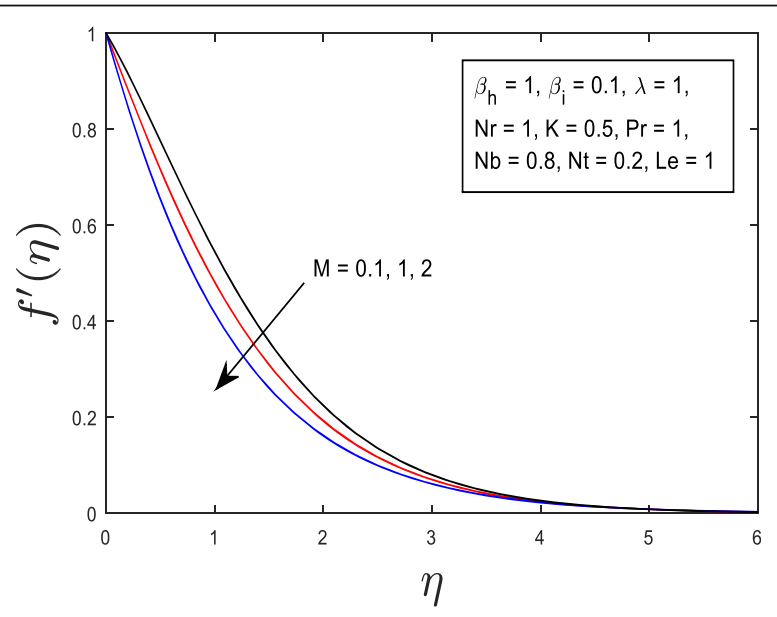

Fig. 2 Graph of velocity for various values of $M$ 


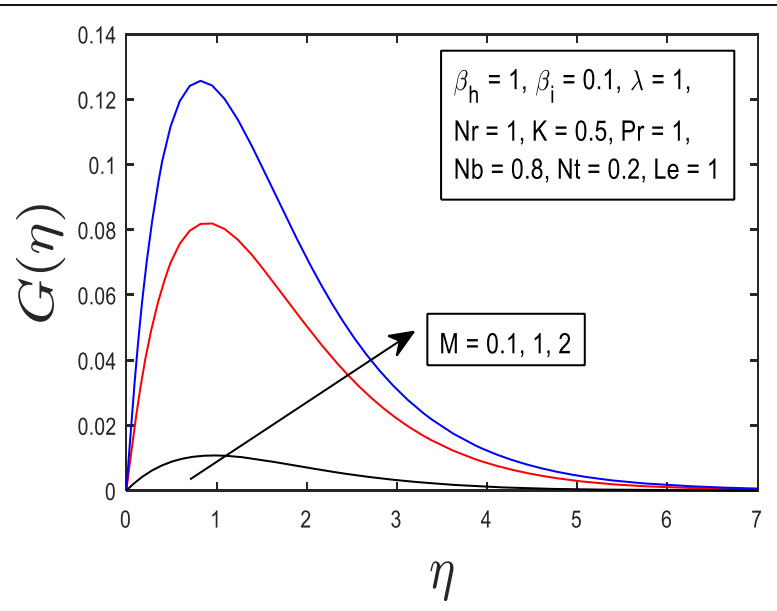

Fig. 3 Graph of cross flow velocity for various values of $M$

$$
\begin{gathered}
u=V_{w}(x)=A x, v=w=0, \\
T=T_{w}(x)=T_{\infty}+B x, \\
\Phi=\Phi_{w}(x)=\Phi_{\infty}+C x \quad \text { at } y=0, \\
u \rightarrow 0, w \rightarrow 0, T \rightarrow T_{\infty}, \\
\Phi \rightarrow \Phi_{\infty}, \quad \text { as } y \rightarrow \infty
\end{gathered}
$$

where $A, C(>0)$ and $B$ are constants, and $T_{w}(x)$ and $\Phi_{w}(x)$ are variable surface temperature and concentration, respectively. Moreover, $B>0$ denotes heated plate and $B<0$ denotes cooled plate.

Let us establish the similarity transformations $([39,43])$

$$
\begin{aligned}
\eta & =\left(\frac{A}{v}\right)^{1 / 2} y, \quad \psi=(A v)^{1 / 2} x f(\eta), \\
w & =A x G(\eta), \quad \theta(\eta)=\frac{T-T_{\infty}}{T_{w}-T_{\infty}} \\
\varphi(\eta) & =\frac{\Phi-\Phi_{\infty}}{\Phi_{w}-\Phi_{\infty}}
\end{aligned}
$$

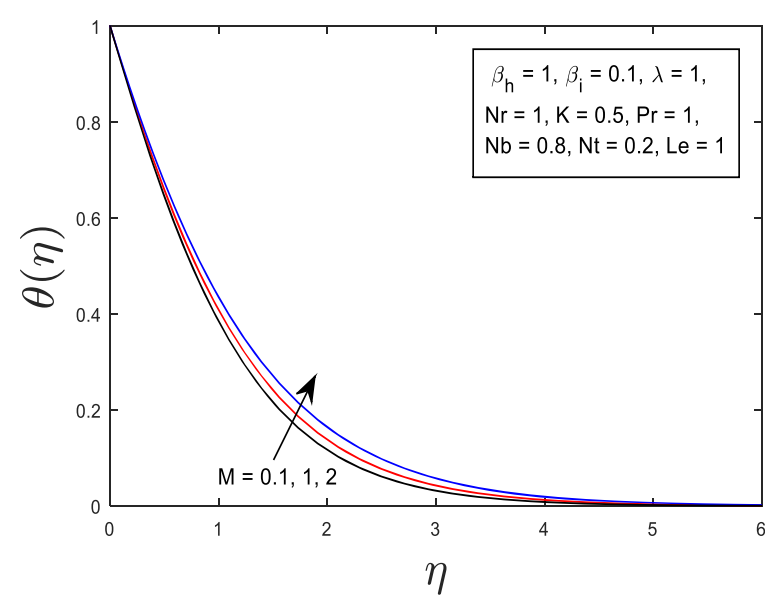

Fig. 4 Graph of temperature for various values of $M$ 


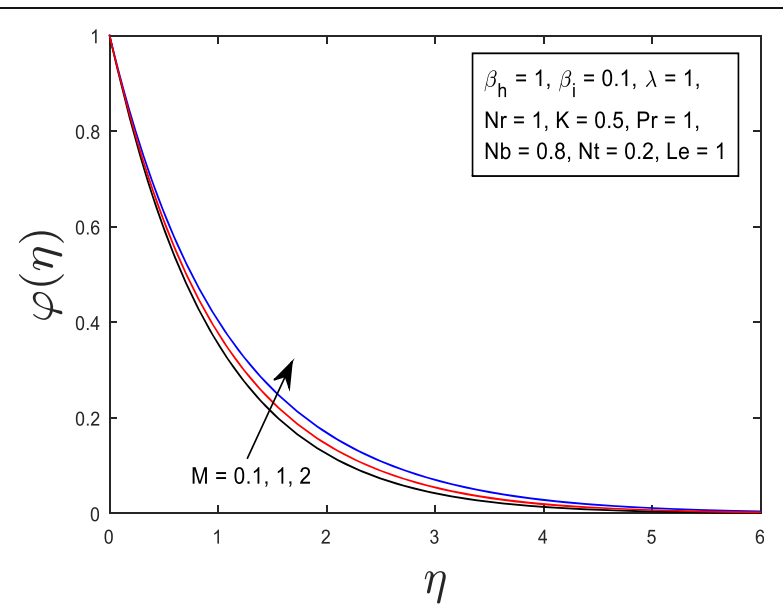

Fig. 5 Graph of concentration for various values of $M$

to obtain similarity solutions of Eqs. (1)-(5) subject to the boundary conditions (6), where the stream function $\psi(x, y)$ is defined as:

$$
u=\frac{\partial \psi}{\partial y}, v=-\frac{\partial \psi}{\partial x}
$$

Substituting Eqs. (7) into Eqs. (2)-(5), the following ordinary differential equations are obtained:

$$
\begin{aligned}
& f^{\prime \prime \prime}+f f^{\prime \prime}-\left(f^{\prime}\right)^{2}-\frac{M}{\left(\beta^{2}+\beta_{h}^{2}\right)}\left(\beta f^{\prime}+\beta_{h} G\right)+\lambda(\theta+N r \varphi)-K f^{\prime}=0 \\
& G^{\prime \prime}+f G^{\prime}-f^{\prime} G+\frac{M}{\left(\beta^{2}+\beta_{h}^{2}\right)}\left(\beta_{h} f^{\prime}-\beta G\right)-K G=0 \\
& \theta^{\prime \prime}+\operatorname{Pr}\left(f \theta^{\prime}-f^{\prime} \theta+N b \theta^{\prime} \varphi^{\prime}+N t \theta^{\prime 2}\right)=0
\end{aligned}
$$

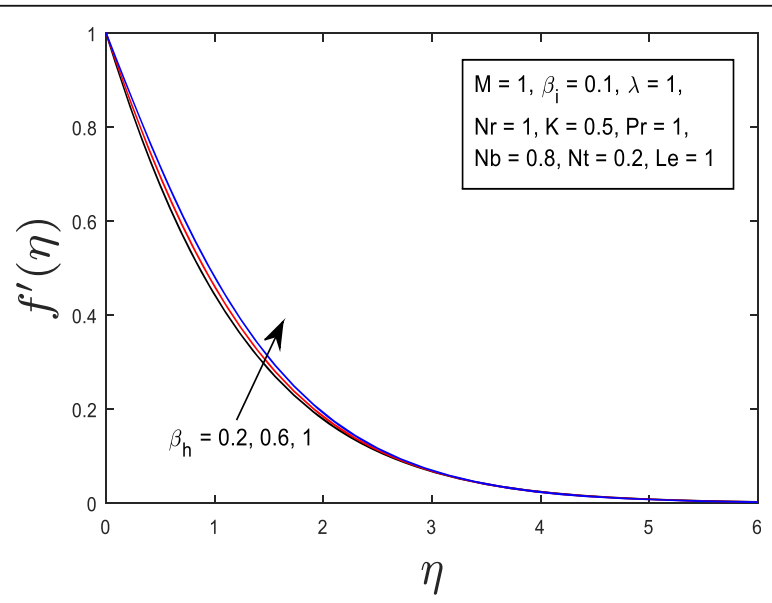

Fig. 6 Graph of velocity for various values of $\beta_{h}$ 


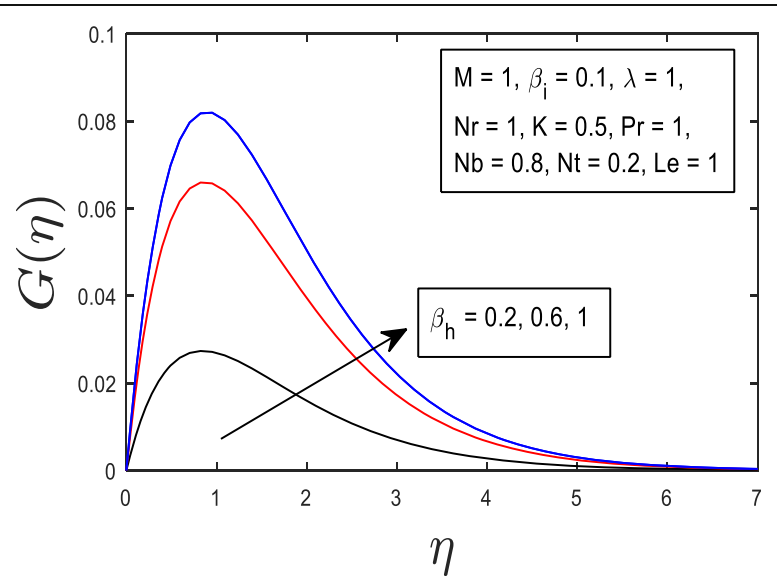

Fig. 7 Graph of cross flow velocity for various values of $\boldsymbol{\beta}_{\boldsymbol{h}}$

$$
\varphi^{\prime \prime}+\operatorname{Pr} L e\left(f \varphi^{\prime}-f^{\prime} \varphi\right)+\frac{N t}{N b} \theta^{\prime \prime}=0
$$

with the transformed boundary conditions:

$$
\begin{gathered}
f(0)=0, \quad f^{\prime}(0)=1, \quad G(0)=0, \\
\theta(0)=1, \quad \varphi(0)=1, \\
f^{\prime} \rightarrow 0, G \rightarrow 0, \theta \rightarrow 0, \\
\varphi \rightarrow 0, \text { as } \quad \eta \rightarrow \infty,
\end{gathered}
$$

where $M=\sigma M_{0}^{2} / \rho A$ is the magnetic parameter, $\lambda=G r_{x} / R e_{x}^{2}$ is the constant mixed convection parameter where $G r_{x}=\mathrm{g} B_{T}\left(T_{w}-T_{\infty}\right) x^{3} / v^{2}$ is the local Grashof number, and $R e_{x}=A x^{2} / v$ is the local Reynolds number, $N r=B_{C}\left(\Phi_{w}-\Phi_{\infty}\right) / B_{T}\left(T_{w}-T_{\infty}\right)$ is the buoyancy ratio, $K=v / A K$ is the permeability parameter, $P r=v / \alpha$ is the Prandtl number, $N b=$ $\gamma D_{B}\left(\Phi_{w}-\Phi_{\infty}\right) / v$ is the Brownian motion parameter, $N t=\gamma D_{T}\left(T_{w}-T_{\infty}\right) / v T_{\infty}$ is the thermophoresis parameter, $L e=\alpha / D_{B}$ is the Lewis number, and $\gamma=(\rho \Phi)_{p} /(\rho \Phi)_{f}$ is the ratio between the heat capacity of the nanoparticle material and heat capacity of the fluid.

The physical quantities of interest are the skin friction coefficients $C_{f x}$ and $C_{f z}$, the local Nusselt number $N u_{x}$, and the Sherwood number $S h_{x}$ which are defined as follows:

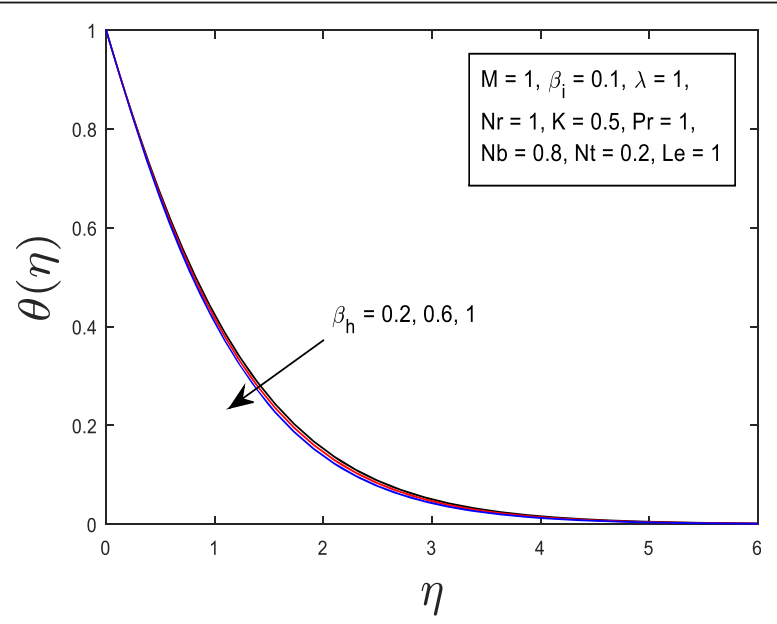

Fig. 8 Graph of temperature for various values of $\beta_{h}$ 


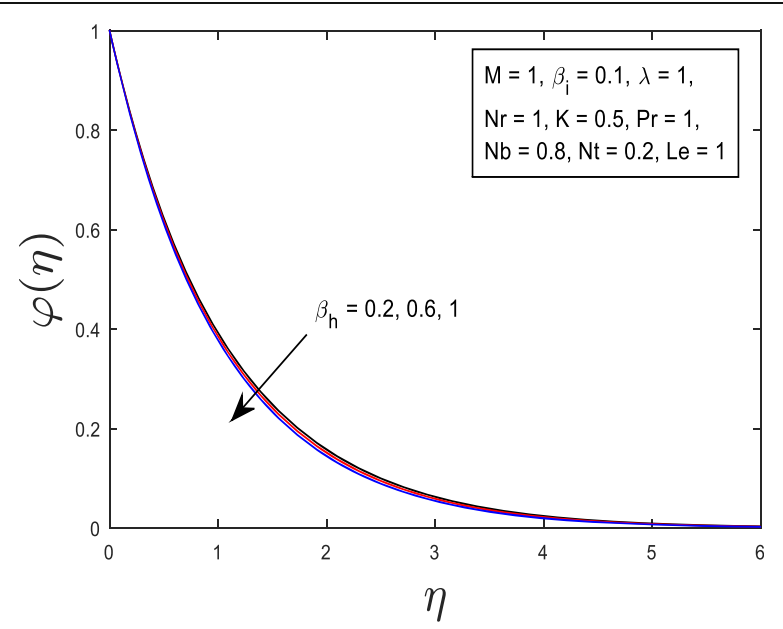

Fig. 9 Graph of concentration for various values of $\boldsymbol{\beta}_{\boldsymbol{h}}$

$$
C_{f x}=\frac{2 \tau_{w x}}{\rho V_{w}^{2}}, \quad C_{f z}=\frac{2 \tau_{w z}}{\rho V_{w}^{2}}, \quad N u_{x}=\frac{x q_{w}}{k\left(T_{W}-T_{\infty}\right)}, \quad S h_{x}=\frac{x j_{m}}{D_{B}\left(\Phi_{W}-\Phi_{\infty}\right)}
$$

where $\tau_{w x}$ and $\tau_{w z}$ are the wall shear stresses in the directions of $x$ and $z$, respectively, $q_{w}$ is the surface heat flux, and $\mathrm{j}_{m}$ is the surface mass flux, which are given by

$$
\begin{gathered}
\tau_{w x}=\left.\mu\left(\frac{\partial u}{\partial y}\right)\right|_{y=0}, \tau_{w z}=\left.\mu\left(\frac{\partial w}{\partial y}\right)\right|_{y=0}, \\
q_{w}=-\left.k\left(\frac{\partial T}{\partial y}\right)\right|_{y=0}, \\
\mathrm{j}_{m}=-\left.D_{B}\left(\frac{\partial \Phi}{\partial y}\right)\right|_{y=0}
\end{gathered}
$$

From Eqs. (7) and (14), we get

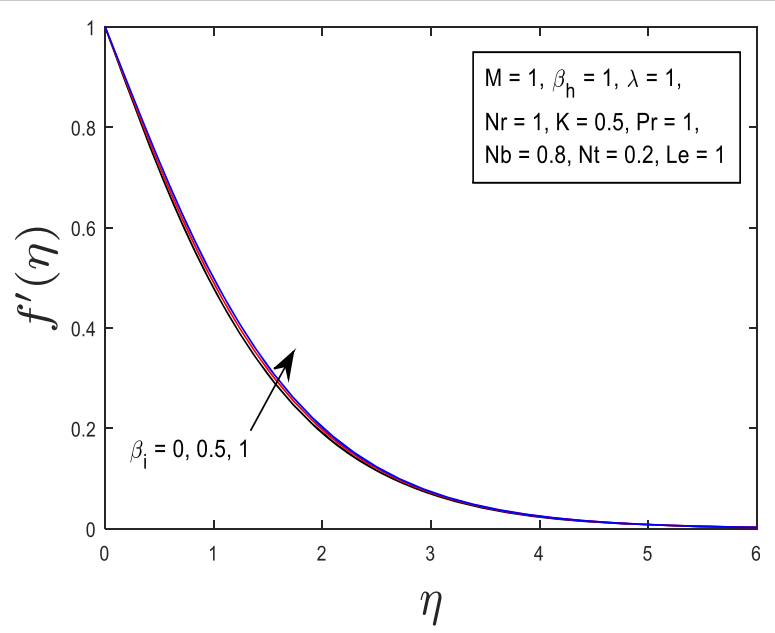

Fig. 10 Graph of velocity for various values of $\boldsymbol{\beta}_{\boldsymbol{i}}$ 


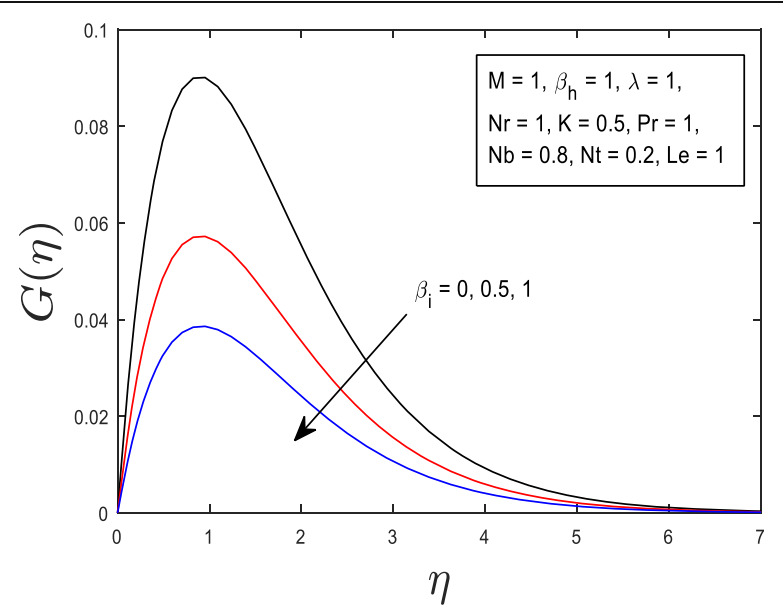

Fig. 11 Graph of cross flow velocity for various values of $\beta_{i}$

$$
\begin{aligned}
C_{f x} & =\frac{2}{\sqrt{\operatorname{Re}_{x}}} f^{\prime \prime}(0), \quad C_{f z}=\frac{2}{\sqrt{\operatorname{Re}_{x}}} G^{\prime}(0), N u_{x}=-\sqrt{\operatorname{Re}_{x}} \theta^{\prime}(0), \\
S h_{x} & =-\sqrt{\operatorname{Re}_{x}} \varphi^{\prime}(0) .
\end{aligned}
$$

\section{Method of solution}

The spectral relaxation method (SRM) is used to solve the system of nonlinear ordinary differential Eqs. (9)-(12) subject to the boundary conditions (13). To implement the SRM, the order of the momentum Eq. (9) is reduced to second order introducing the transformation $f^{\prime}=H$ so that $f^{\prime \prime}=H^{\prime}$ and $f=H^{\prime \prime}$. Hence, Eqs. (9)-(12) and the boundary conditions (13) can be transformed as follows:

$$
f^{\prime}=H
$$

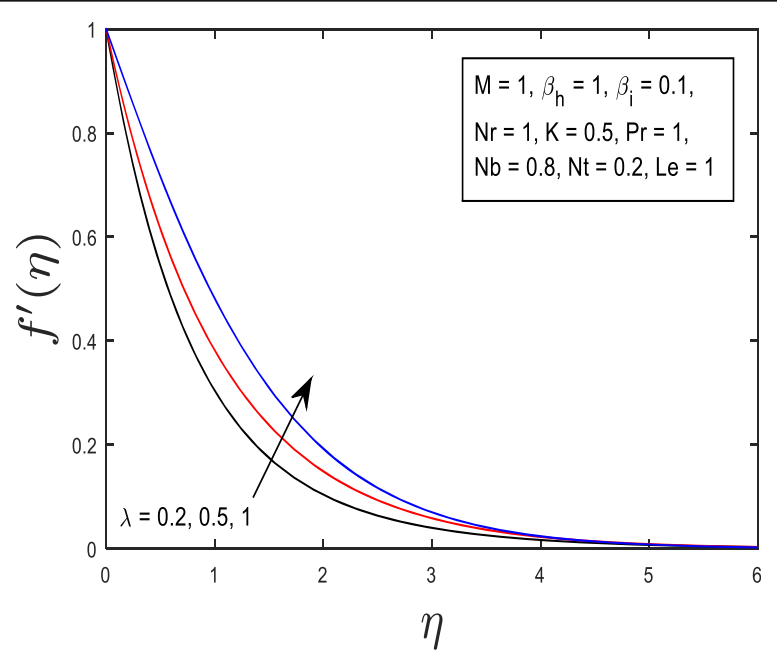

Fig. 12 Graph of velocity for various values of $\boldsymbol{\lambda}$ 


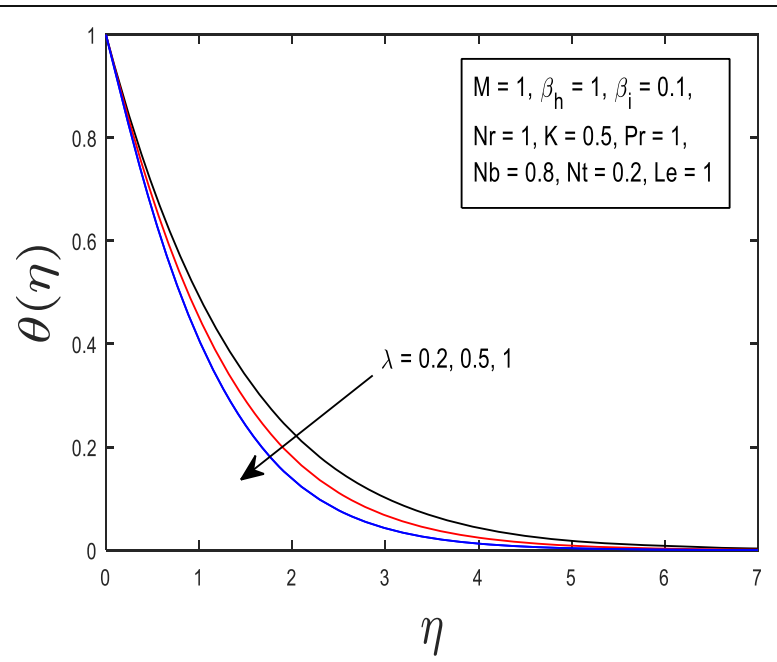

Fig. 13 Graph of temperature for various values of $\boldsymbol{\lambda}$

$$
\begin{aligned}
& H^{\prime \prime}+f H^{\prime}-(H)^{2}-\frac{M}{\left(\beta^{2}+\beta_{h}^{2}\right)}\left(\beta H+\beta_{h} G\right)+\lambda(\theta+N r \varphi)-K H=0 \\
& G^{\prime \prime}+f G^{\prime}-H G+\frac{M}{\left(\beta^{2}+\beta_{h}^{2}\right)}\left(\beta_{h} H-\beta G\right)-K G=0 \\
& \theta^{\prime \prime}+\operatorname{Pr}\left(f \theta^{\prime}-H \theta+N b \theta^{\prime} \varphi^{\prime}+N t \theta^{2}\right)=0 \\
& \varphi^{\prime \prime}+\operatorname{PrLe}\left(f \varphi^{\prime}-H \varphi\right)+\frac{N t}{N b} \theta^{\prime \prime}=0
\end{aligned}
$$

subject to the boundary conditions

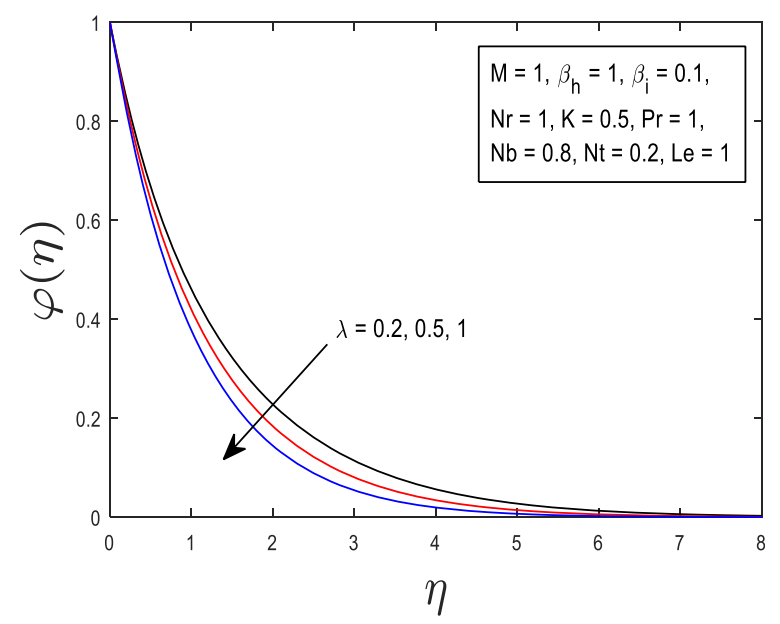

Fig. 14 Graph of concentration for various values of $\boldsymbol{\lambda}$ 


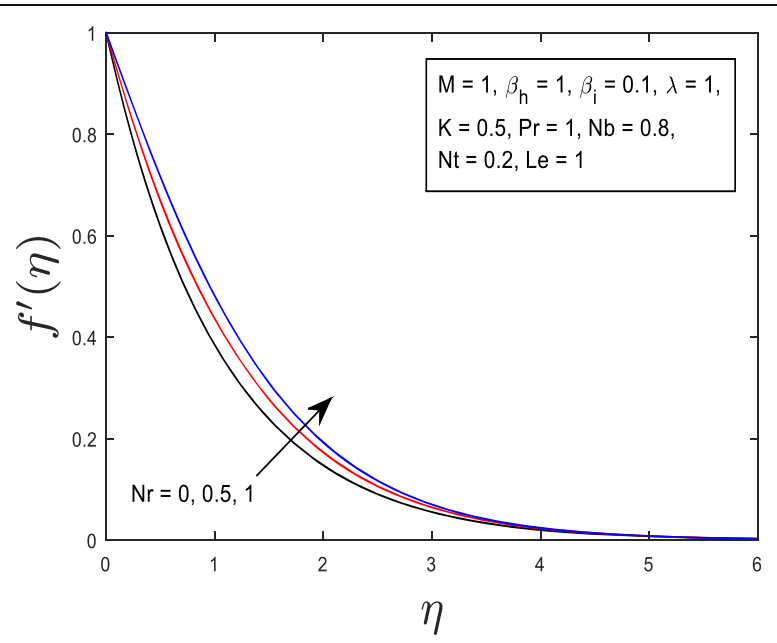

Fig. 15 Graph of velocity for various values of $\mathrm{Nr}$

$$
\begin{gathered}
f(0)=0, H(0)=1, G(0)=0 \\
\theta(0)=1, \quad \varphi(0)=1, \\
H \rightarrow 0, G \rightarrow 0, \theta \rightarrow 0, \varphi \rightarrow 0 \\
\text { as } \eta \rightarrow \infty .
\end{gathered}
$$

Detail rules of the SRM can be found in ([40-42]). The resulting iteration scheme in SRM for Eqs. (17)-(22) can be written as follows:

$$
\begin{aligned}
& f_{r+1}^{\prime}=H_{r} \\
& H_{r+1}^{\square}+f_{r+1} H_{r+1}^{\prime}-H_{r}^{2}-\frac{M}{\left(\beta^{2}+\beta_{h}^{2}\right)}\left(\beta H_{r+1}+\beta_{h} G_{r}\right)+\lambda\left(\theta_{r}+N r \varphi_{r}\right)-K H_{r+1}=0 \\
& G_{r+1}^{\prime \prime}+f_{r+1} G_{r+1}^{\prime}-H_{r+1} G_{r+1}+\frac{M}{\left(\beta^{2}+\beta_{h}^{2}\right)}\left(\beta_{h} H_{r+1}-\beta G_{r+1}\right)-K G_{r+1}=0
\end{aligned}
$$

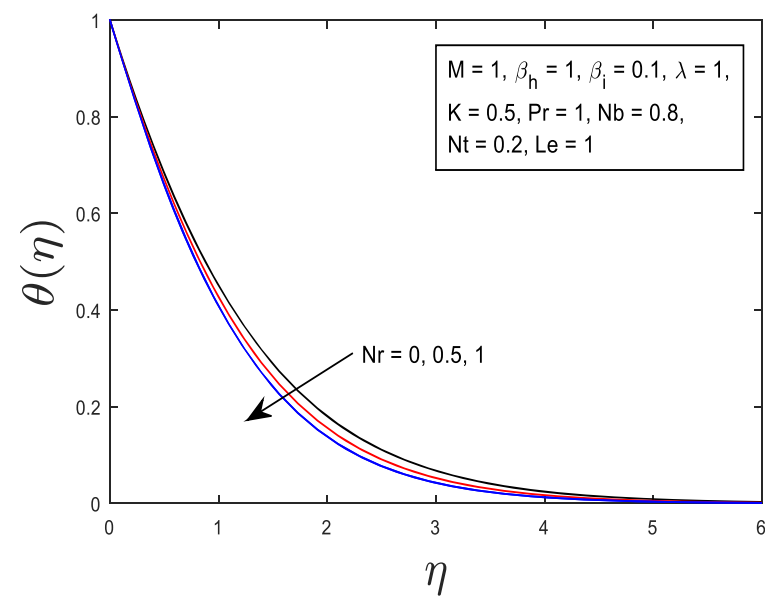

Fig. 16 Graph of temperature for various values of $\mathrm{Nr}$ 


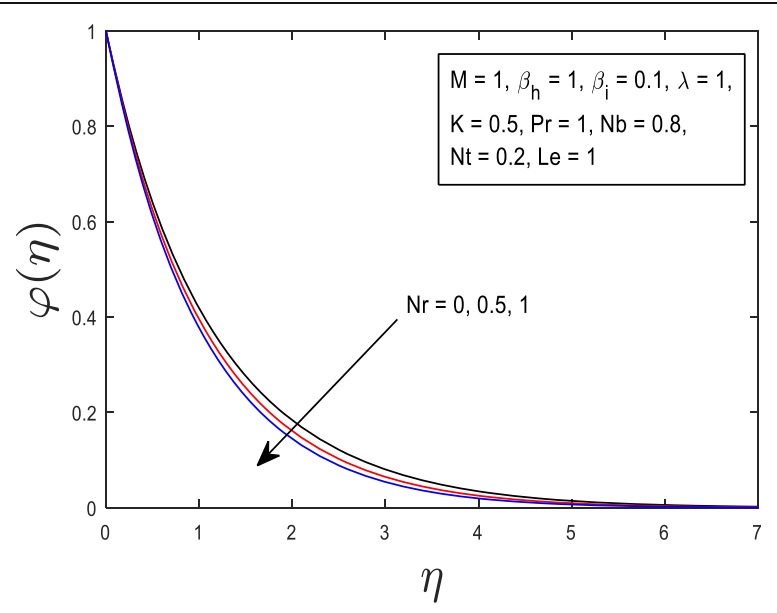

Fig. 17 Graph of concentration for various values of $\mathrm{Nr}$

$$
\begin{aligned}
& \theta_{r+1}^{\prime \prime}+\operatorname{Pr}\left(f_{r+1} \theta_{r+1}^{\prime}-H_{r+1} \theta_{r+1}+N b \theta_{r+1}^{\prime} \varphi_{r}^{\prime}+N t \theta_{r}^{\prime 2}\right)=0 \\
& \varphi_{r+1}^{\prime \prime}+\operatorname{PrLe}\left(f_{r+1} \varphi_{r+1}^{\prime}-H_{r+1} \varphi_{r+1}\right)+\frac{N t}{N b} \theta_{r+1}^{\prime \prime}=0
\end{aligned}
$$

subject to the boundary conditions

$$
\begin{gathered}
f_{r+1}(0)=0, H_{r+1}(0)=1, G_{r+1}(0)=0, \\
\theta_{r+1}(0)=1, \quad \varphi_{r+1}(0)=1, \\
H_{r+1} \rightarrow 0, \quad G_{r+1} \rightarrow 0, \theta_{r+1} \rightarrow 0, \\
\varphi_{r+1} \rightarrow 0 \text { as } \eta \rightarrow \infty .
\end{gathered}
$$

In Eqs. (23)-(28), $r+1$ and $r$ denote the current and previous iteration, respectively. Implementing Chebyshev differentiation (Trefethen [44]) on iterative scheme defined by Eqs. (23)-(27), we obtain the following discrete form:

$$
\begin{array}{ll}
\boldsymbol{B}_{\mathbf{1}} \mathbf{f}_{r+1}=\boldsymbol{R}_{\mathbf{1}}, & f_{r+1}\left(\zeta_{N}\right)=0 \\
\boldsymbol{B}_{\mathbf{2}} \mathbf{H}_{r+1}=\boldsymbol{R}_{\mathbf{2}}, & H_{r+1}\left(\zeta_{N}\right)=1, \quad H_{r+1}\left(\zeta_{0}\right)=0
\end{array}
$$

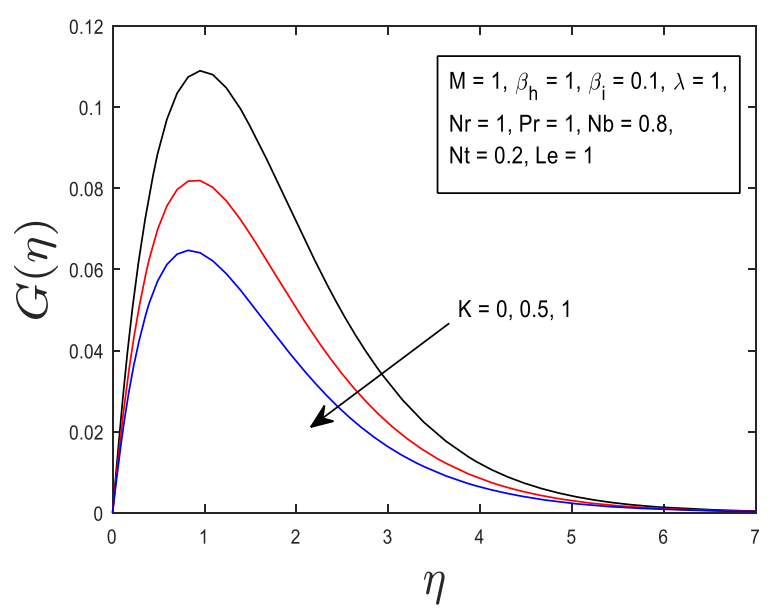

Fig. 18 Graph of cross flow profiles for various values of $K$ 


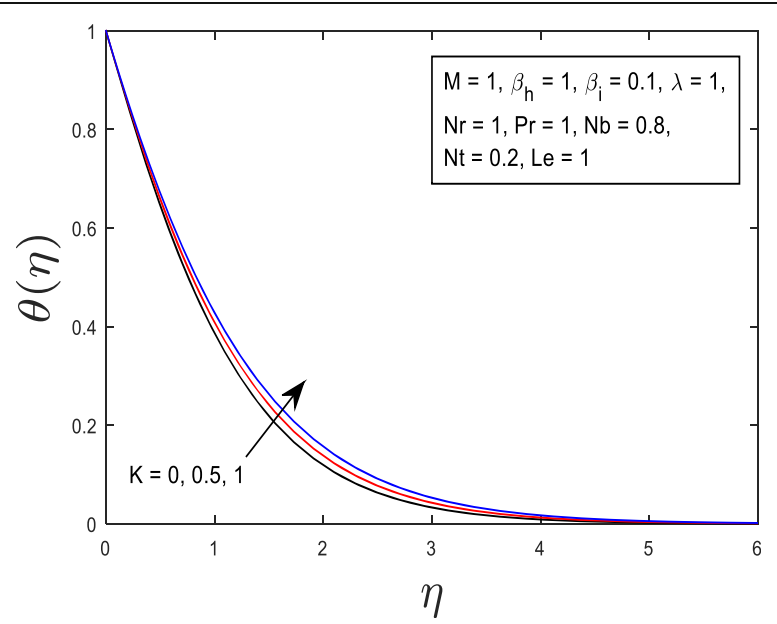

Fig. 19 Graph of temperature for various values of $K$

$$
\begin{aligned}
& \boldsymbol{B}_{\mathbf{3}} \mathbf{G}_{\mathbf{r}+1}=\mathbf{R}_{\mathbf{3}}, \quad G_{r+1}\left(\zeta_{N}\right)=0, \quad G_{r+1}\left(\zeta_{0}\right)=0 \\
& \boldsymbol{B}_{\mathbf{4}} \boldsymbol{\theta}_{\mathbf{r}+1}=\mathbf{R}_{\mathbf{4}}, \quad \theta_{r+1}\left(\zeta_{N}\right)=1, \theta_{r+1}\left(\zeta_{0}\right)=0 \\
& \boldsymbol{B}_{\mathbf{5}} \boldsymbol{\phi} \_\mathbf{r}+1=\mathbf{R}_{\mathbf{5}}, \quad \varphi_{r+1}\left(\zeta_{N}\right)=1, \quad \varphi_{r+1}\left(\zeta_{0}\right)=0
\end{aligned}
$$

where

$$
\begin{gathered}
\boldsymbol{B}_{\mathbf{1}}=\boldsymbol{D}, \quad \quad \boldsymbol{R}_{-} \mathbf{1}=H_{r} \\
B_{2}=D^{2}+\operatorname{diag}\left(f_{r+1}\right) D-\left(\frac{M \beta}{\beta^{2}+\beta_{h}^{2}}+K\right) \mathbf{I}, \\
\quad \mathbf{R} \_2=H_{r}^{2}+\frac{M \beta_{h}}{\left(\beta^{2}+\beta_{h}^{2}\right)} G_{r}-\lambda\left(\theta_{r}+N r \varphi_{r}\right)
\end{gathered}
$$

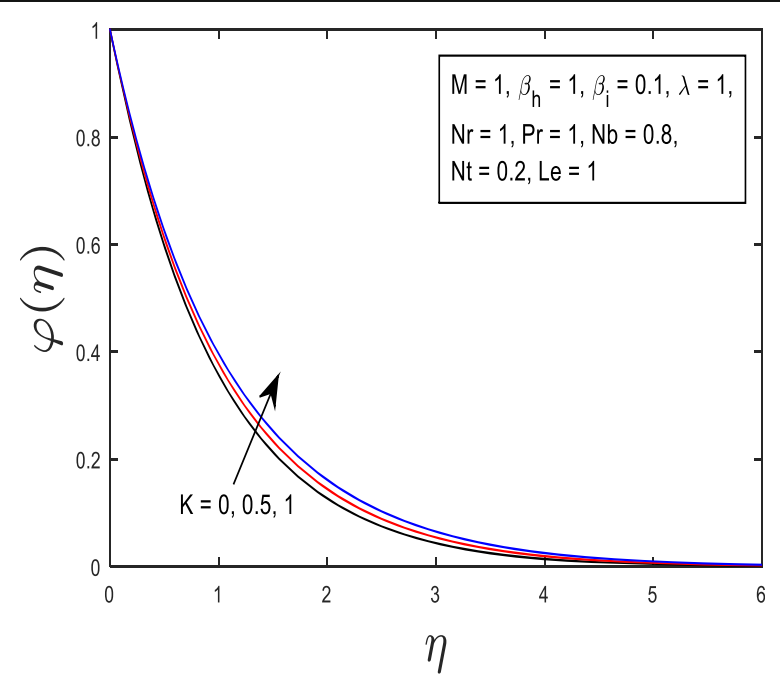

Fig. 20 Graph of concentration for various values of $K$ 


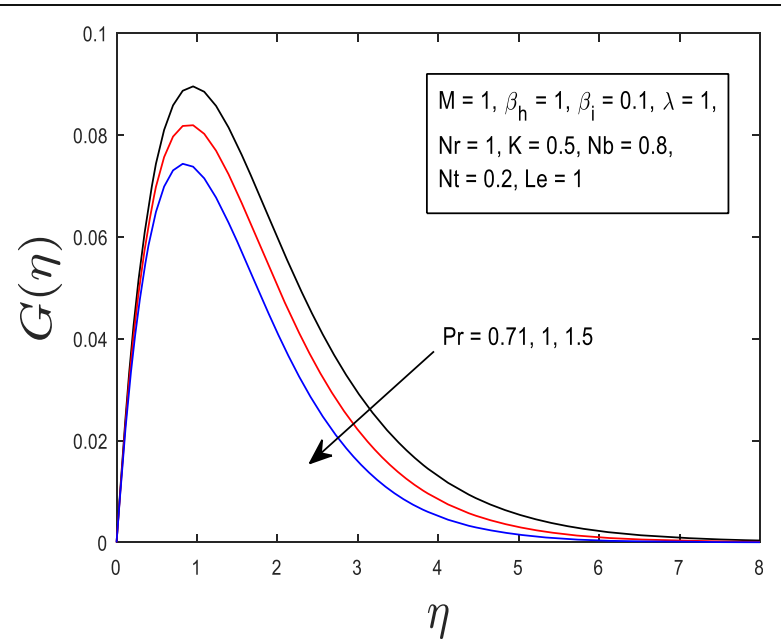

Fig. 21 Graph of cross flow velocity for various values of $\mathrm{Pr}$

$$
\begin{aligned}
& B_{3}=D^{2}+\operatorname{diag}\left(f_{r+1}\right) D-\operatorname{diag}\left(H_{r+1}\right)-\left(\frac{M \beta}{\beta^{2}+\beta_{h}^{2}}+K\right) \mathbf{I}, \\
& R_{\_} 3=-\frac{M \beta_{h}}{\beta^{2}+\beta_{h}^{2}} H_{r+1} \\
& B_{4}=D^{2}+\operatorname{Pr} \operatorname{diag}\left(f_{r+1}+N b \varphi_{r}^{\prime}\right) D-\operatorname{Pr} \operatorname{diag}\left(H_{r+1}\right), \\
& R_{-} 4=-P r N t \theta_{r}^{\prime 2} \\
& B_{5}=D^{2}+\operatorname{Le}\left(\operatorname{diag}\left(f_{r+1}\right) D-L e \operatorname{diag}\left(H_{r+1}\right),\right. \\
& R_{-} 5=-\frac{N t}{N b} \theta_{r+1}^{\prime \prime}
\end{aligned}
$$

where $\boldsymbol{D}=2 D / \eta_{\infty}, D$ is the Chebyshev differentiation matrix, and $\mathbf{I}$ is the identity matrix of size $(N+1) \times(N+1)$. The decoupled Eqs. (29)-(33) can be solved independently by choosing a proper initial guesses:

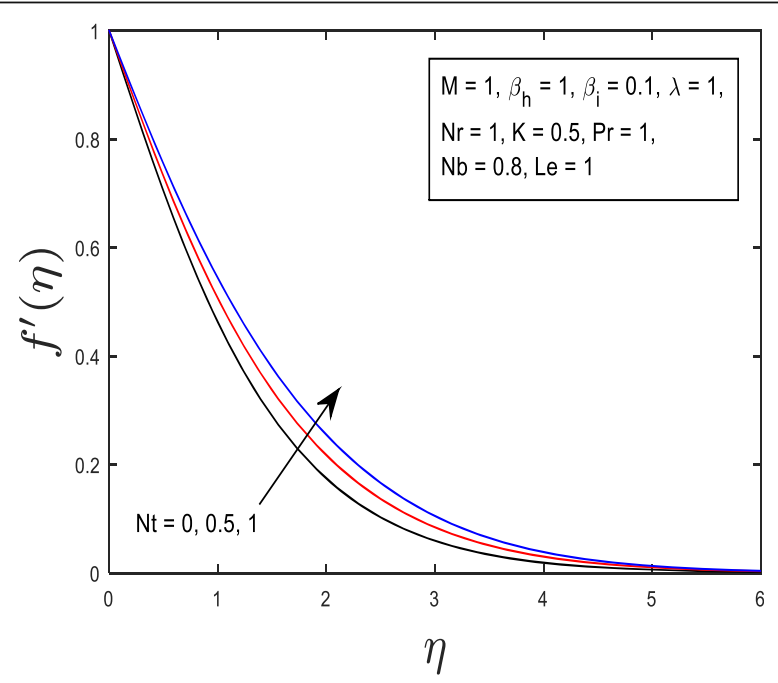

Fig 22 Graph of velocity for various values of $\mathrm{Nt}$ 


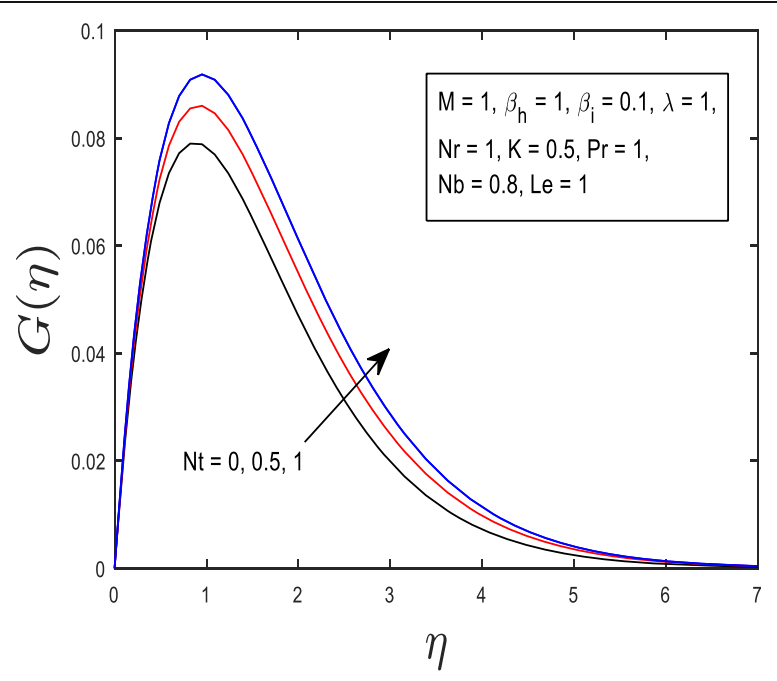

Fig. 23 Graph of cross flow velocity for various values of $\mathrm{Nt}$

$$
\begin{gathered}
f_{0}(\eta)=1-\exp (-\eta), \\
G_{0}(\eta)=\eta \exp (-\eta) \\
\theta_{0}(\eta)=\phi_{-} 0(\eta)=\exp (-\eta)
\end{gathered}
$$

\section{Results and discussions}

The nonlinear ordinary differential Eqs. (9) to (12) with respect to the boundary conditions (13) are solved numerically using SRM. The numerical solutions are obtained for velocities, temperature, and concentration profiles for different values of governing parameters. The graphical representations of the numerical results are shown in Figs. 2, 3, $4,5,6,7,8,9,10,11,12,13,14,15,16,17,18,19,20,21,22$ and 23. Unless otherwise stated, the numerical values for the parameters are taken to be fixed as $M=\beta_{h}=\lambda=$ $N r=P r=L e=1.0, \beta_{i}=0.1, K=0.5, \quad N b=0.8$, and $N t=0.2$.

The ranges of parameters used in the figures are $0.1 \leq M \leq 2.0,0.2 \leq \beta_{h} \leq 1.0$, $0.0 \leq \beta_{i} \leq 1.5, \quad 0.2 \leq \lambda \leq 1.0, \quad 0.0 \leq N r \leq 1.0,0.0 \leq K \leq 1.0,0.71 \leq P r \leq 1.5$, and $0.0 \leq N t \leq$ 1.0. For the authentication of the numerical method used, the results were compared with the previously obtained results for various values of parameters and it indicates an excellent accord as shown in Tables 1 and 2.

From Table 3, it is observed that the skin friction coefficient $-f^{\prime \prime}(0)$ in the $x$-direction increases with the increasing values of $\beta_{h}, \beta_{i}, \lambda, N r$, and $N t$, and it decreases for

Table 1 Comparison of $-\theta^{\prime}(0)$ for various values of $\operatorname{Pr}$ when $M=\beta_{h}=\beta_{i}=\lambda=\mathrm{Nr}=K=\mathrm{Nb}=\mathrm{Nt}=$ Le $=\varphi=0$

\begin{tabular}{lllll}
\hline $\operatorname{Pr}$ & Ali et al. [39] & Ramzan et al. [43] & Grubka and Bobba [45] & Present results \\
\hline 0.01 & 0.0198 & - & 0.0197 & 0.01988750 \\
0.72 & 0.8086 & 0.8086313 & 0.8086 & 0.80863151 \\
1 & 1.0000 & 1.0000000 & 1.0000 & 1.00000000 \\
3 & 1.9237 & 1.9359130 & 1.9237 & 1.92368259 \\
10 & 3.7208 & 3.7215958 & 3.7207 & 3.72067428 \\
100 & 12.3004 & - & 12.2940 & 12.29413922 \\
\hline
\end{tabular}


Table 2 Comparison of $-f^{\prime \prime}(0)$ and $-\theta^{\prime}(0)$ for different values of $M$ with $\beta_{h}=0,2, \lambda=\operatorname{Pr}=1$ and $\beta_{i}=\mathrm{Nr}=K=\mathrm{Nb}=\mathrm{Nt}=\mathrm{Le}=\varphi=0$

\begin{tabular}{|c|c|c|c|c|c|c|c|c|}
\hline \multirow[t]{3}{*}{ M } & \multicolumn{4}{|c|}{ Ali et al. [39] } & \multicolumn{4}{|c|}{ Present results } \\
\hline & \multicolumn{2}{|l|}{$\overline{\beta_{h}=0}$} & \multicolumn{2}{|l|}{$\beta_{h}=2$} & \multicolumn{2}{|l|}{$\beta_{h}=0$} & \multicolumn{2}{|l|}{$\beta_{h}=2$} \\
\hline & $-f^{\prime \prime}(0)$ & $-\theta^{\prime} \quad(0)$ & $-f^{\prime \prime}(0)$ & $-\theta^{\prime} \quad(0)$ & $-f^{\prime \prime}(0)$ & $-\theta^{\prime} \quad(0)$ & $-f^{\prime \prime}(0)$ & $-\theta^{\prime}(0)$ \\
\hline 0 & 0.5607 & 1.0873 & 0.5608 & 1.0873 & 0.560941 & 1.087206 & 0.560941 & 1.087206 \\
\hline 0.01 & 0.5658 & 1.0863 & 0.5618 & 1.0871 & 0.565820 & 1.086269 & 0.561782 & 1.087072 \\
\hline 0.04 & 0.5810 & 1.0833 & 0.5649 & 1.0865 & 0.581027 & 1.083262 & 0.564835 & 1.086452 \\
\hline 0.25 & 0.6830 & 1.0630 & 0.5878 & 1.0816 & 0.683029 & 1.063010 & 0.587819 & 1.081601 \\
\hline 1 & 1.0000 & 1.0000 & 0.6816 & 1.0591 & 1.000000 & 1.000000 & 0.681587 & 1.059134 \\
\hline
\end{tabular}

increasing values of $M$ and $\mathrm{Nb}$. It is also noticed that the skin friction coefficient $G$

(0) in the $z$-direction increases as $M, \beta_{h}, \lambda, N r$, and $N t$ increase, and it decreases by increasing $\beta_{i}$ and $N b$. The table also shows that the local Nusselt number $-\theta^{\prime} \quad(0)$ increases with an increase in $\beta_{h}, \beta_{i}, \lambda$, and $N r$, and it decreases with an increase in $M, N b$, and $N t$. Furthermore, the local Sherwood number $-\varphi^{\prime}(0)$ increases as $\beta_{h}, \beta_{i}, \lambda, N r$, and $N b$ increase and decreases as $M$ and $N t$ increase.

Figures 2, 3, 4 and 5 show the effects of magnetic parameter $M$ on the velocity $f^{\prime}(\eta)$, cross flow velocity $G(\eta)$, temperature $\theta(\eta)$, and concentration $\varphi(\eta)$ profiles, respectively. The velocity profile $f^{\prime}(\eta)$ decreases with an increase in the values of $M$, whereas the cross

Table 3 Numerical values of the skin friction coefficients $-f^{\prime \prime}(0)$ and $G^{\prime}(0)$, local Nusselt number $-\theta^{\prime}(0)$, and local Sherwood number $-\varphi^{\prime}$ (0) for various values of $M, \beta_{h}, \beta_{i}, \lambda, N r, N b$, and $N t$ when $K=0.5, \operatorname{Pr}=1$, and $L e=1$ are fixed

\begin{tabular}{llllllllllll}
\hline$M$ & $\beta_{h}$ & $\beta_{i}$ & $\lambda$ & $N r$ & $N b$ & $N t$ & $-f^{\prime \prime}(0)$ & $G^{\prime}(0)$ & $-\theta^{\prime}(0)$ & $-\varphi^{\prime}(0)$ \\
\hline 0.0 & 1.0 & 0.1 & 1.0 & 1.0 & 0.8 & 0.2 & 0.358052 & 0.000000 & 0.787875 & 1.022604 \\
0.2 & 1.0 & 0.1 & 1.0 & 1.0 & 0.8 & 0.2 & 0.404568 & 0.054074 & 0.783140 & 1.014387 \\
0.4 & 1.0 & 0.1 & 1.0 & 1.0 & 0.8 & 0.2 & 0.451324 & 0.104165 & 0.778270 & 1.005904 \\
1.0 & 0.0 & 0.1 & 1.0 & 1.0 & 0.8 & 0.2 & 0.774312 & 0.000000 & 0.745503 & 0.950242 \\
1.0 & 0.3 & 0.1 & 1.0 & 1.0 & 0.8 & 0.2 & 0.738252 & 0.121134 & 0.748909 & 0.955825 \\
1.0 & 0.6 & 0.1 & 1.0 & 1.0 & 0.8 & 0.2 & 0.674238 & 0.196848 & 0.754994 & 0.965852 \\
1.0 & 1.0 & 0.0 & 1.0 & 1.0 & 0.8 & 0.2 & 0.594820 & 0.258345 & 0.762577 & 0.978417 \\
1.0 & 1.0 & 0.1 & 1.0 & 1.0 & 0.8 & 0.2 & 0.590784 & 0.234345 & 0.763224 & 0.979652 \\
1.0 & 1.0 & 0.2 & 1.0 & 1.0 & 0.8 & 0.2 & 0.585817 & 0.212930 & 0.763922 & 0.980946 \\
1.0 & 1.0 & 0.1 & 0.0 & 1.0 & 0.8 & 0.2 & 1.424897 & 0.174459 & 0.649559 & 0.776368 \\
1.0 & 1.0 & 0.1 & 0.2 & 1.0 & 0.8 & 0.2 & 1.236853 & 0.192259 & 0.685824 & 0.845391 \\
1.0 & 1.0 & 0.1 & 0.5 & 1.0 & 0.8 & 0.2 & 0.981128 & 0.211193 & 0.721370 & 0.908559 \\
1.0 & 1.0 & 0.1 & 1.0 & 0.0 & 0.8 & 0.2 & 0.973008 & 0.211529 & 0.722476 & 0.909963 \\
1.0 & 1.0 & 0.1 & 1.0 & 0.2 & 0.8 & 0.2 & 0.893654 & 0.216702 & 0.731814 & 0.926210 \\
1.0 & 1.0 & 0.1 & 1.0 & 0.4 & 0.8 & 0.2 & 0.815937 & 0.221519 & 0.7404477 & 0.941069 \\
1.0 & 1.0 & 0.1 & 1.0 & 1.0 & 0.1 & 0.2 & 0.459690 & 0.251479 & 1.027856 & -0.019822 \\
1.0 & 1.0 & 0.1 & 1.0 & 1.0 & 0.4 & 0.2 & 0.582084 & 0.236050 & 0.889731 & 0.837193 \\
1.0 & 1.0 & 0.1 & 1.0 & 1.0 & 0.8 & 0.2 & 0.590791 & 0.234344 & 0.763222 & 0.979651 \\
1.0 & 1.0 & 0.1 & 1.0 & 1.0 & 0.8 & 0.0 & 0.616011 & 0.230279 & 0.788958 & 1.067908 \\
1.0 & 1.0 & 0.1 & 1.0 & 1.0 & 0.8 & 0.2 & 0.590791 & 0.234344 & 0.763222 & 0.979651 \\
1.0 & 1.0 & 0.1 & 1.0 & 1.0 & 0.8 & 0.4 & 0.567447 & 0.238062 & 0.738055 & 0.903053 \\
\hline & & & & & & & & & &
\end{tabular}


flow velocity $G(\eta)$, temperature $\theta(\eta)$ and concentration $\varphi(\eta)$ profiles increase as $M$ increases. As $M$ increases, a drag force, called Lorentz force increases. Since this force opposes the flow of nanofluid, velocity in the flow direction decreases. Moreover, since an electrically conducting nanofluid with the strong magnetic field in the direction orthogonal to the flow are considered, an increase in $M$ increases the force in the $z$-direction which results in an increase in the cross flow velocity profile $G(\eta)$.

Figures 6, 7, 8 and 9 illustrate the impacts of the Hall parameter $\beta_{h}$ on the velocity $f^{\prime}(\eta)$, cross flow velocity $G(\eta)$, temperature $\theta(\eta)$, and concentration $\varphi(\eta)$ profiles, respectively. It is observed that both the velocity $f^{\prime}(\eta)$ ) and cross flow velocity $G(\eta)$ profiles increase as $\beta_{h}$ increases. But, the temperature and concentration profiles decrease with an increase in $\beta_{h}$ as shown in Figs. 8 and 9. This is because the enclosure of Hall parameter decreases the resistive force caused by the magnetic field due to its effect of reducing the effective conductivity. Hence, the velocity component increases as the Hall parameter increases.

The influences of the ion-slip parameter $\beta_{i}$ on the velocity $f^{\prime}(\eta)$ and cross flow velocity $G(\eta)$ profiles are depicted in Figs. 10 and 11, respectively. The effective conductivity increases with an increase in ion-slip parameter, consecutively damping force decreases on the velocity component in the direction of the flow, and hence, the velocity component increases in the flow direction. Thus, Fig. 10 shows that the velocity profile $f^{\prime}(\eta)$ increases insignificantly as $\beta_{i}$ increases. Conversely, in Fig. 11, a rise in $\beta_{i}$ leads to a decline in the cross flow velocity profile $G(\eta)$.

Figures 12, 13 and 14 disclose the impacts of mixed convection parameter $\lambda$ on the velocity $f^{\prime}(\eta)$, temperature $\theta(\eta)$, and concentration $\varphi(\eta)$ profiles. It is deducted that as $\lambda$ enhances, the velocity profile $f^{\prime}(\eta)$ rises and conversely, the temperature $\theta(\eta)$ and concentration $\varphi(\eta)$ profiles diminish. This is due to the fact that a rise in $\lambda(>0)$ accelerates the fluid's flow for $g>0$ and hence results in an increase in fluid's velocity. Furthermore, fluids having larger value of $\lambda$ have lower thermal diffusivity which reduces the energy capability and the thermal boundary layer thickness.

The effects of the buoyancy ratio $N r$ on the velocity $f^{\prime}(\eta)$, temperature $\theta(\eta)$, and the concentration $\varphi(\eta)$ profiles are shown in Figs. 15, 16 and 17, respectively. Figure 15 reveals that the velocity profile $f^{\prime}(\eta)$ increases as $N r$ increases, while in Figs. 16 and 17, the temperature and concentration $\varphi(\eta)$ profiles decrease as $N r$ increases. The fact is that a rise in $\mathrm{Nr}$ decreases fluid's viscosity. Hence, the result follows.

Figures 18, 19 and 20 demonstrate the effects of the permeability parameter $K$ on the cross flow velocity $G(\eta)$, temperature $\theta(\eta)$, and concentration $\varphi(\eta)$ profiles, respectively. From Fig. 18, it is noticed that as $K$ rises, the cross flow velocity profile diminishes. A rise in resistance against the fluid flow is examined by increasing the thickness of permeable medium which results in a decrease in fluid velocity. In addition, a rise in permeability parameter enhances the thickness of the boundary layer of temperature and concentration. Thus, the temperature and concentration profiles enhance with an increase in $K$ as shown in the Figs. 19 and 20.

Figure 21 divulges the effects of the prandtl number $\operatorname{Pr}$ on the cross flow velocity profile $G(\eta)$. Since fluids having a larger Pr have lower thermal diffusivity, the cross flow velocity profile $G(\eta)$ decreases as the values of Pr increases.

The influence of the thermophoresis parameter $N t$ on the velocity $f^{\prime}(\eta)$ and cross flow velocity $G(\eta)$ profiles are depicted in Figs. 22 and 23, respectively. As it is observed, both flow velocity and cross flow velocity profiles increase as $N t$ increases. This is for the reason 
that the temperature gradient generates a thermophoresis force, and it creates a fast flow away from the stretching sheet. Hence, the velocity profiles increase with an increase in Nt.

\section{Conclusions}

A numerical study of a steady mixed convection flow of an electrically conducting nanofluid over a linearly stretching sheet in the presence of Hall and ion-slip effects has been carried out. With the help of similarity transformations the governing partial differential equations for momentum, energy, and concentration equations were reduced to couple nonlinear ordinary differential equations which were then solved numerically using spectral relaxation method. The effects of different governing parameters on the velocities, temperature, and concentration profiles are studied and revealed the following results:

* The velocity profile $f^{\prime}(\eta)$ increases with an increase in $\beta_{h}, \beta_{i}, \lambda, \mathrm{Nr}$, and $\mathrm{Nt}$ and decreases with an increase in $M$.

* As $M, \beta_{h}$, and Nt increase, the cross flow velocity profile $G(\eta)$ increases.

* As $\beta_{i}, K$, and $\operatorname{Pr}$ increase, the cross flow velocity profile $G(\eta)$ decreases.

* The temperature profile $\theta(\eta)$ increases as $M$ and $K$ increase and decreases as $\beta_{h}, \lambda$, and $\mathrm{Nr}$ increase.

* The concentration profile $\varphi(\eta)$ increases as $M$ and $K$ increase and decreases with an increase in $\beta_{h}, \lambda$, and $\mathrm{Nr}$.

\section{Nomenclature}

$A, B, C$ Constants

$x, y, z$ Cartesian coordinates

$u, v, w$ Velocity components

$V_{w}(x)$ Velocity of the stretching sheet

$M_{0}$ Constant magnetic field strength

g Acceleration due to gravity

$T$ Fluid temperature

$T_{w}$ Surface temperature

$T_{\infty}$ Ambient temperature

$\Phi$ Concentration of fluid

$\Phi_{w}$ Surface concentration

$\Phi_{\infty}$ Ambient concentration

$\mu$ Dynamic viscosity of the fluid

$\rho$ Density of fluid

$v$ Kinematic viscosity of the fluid

$\lambda$ Mixed convection parameter

$\alpha$ Thermal diffusivity

$\sigma$ Electrical conductivity

$\beta_{h}$ Hall parameter

$\beta_{i}$ Ion-slip parameter

$\mathrm{B}_{T}$ Solutal expansion coefficient

$B_{C}$ Thermal expansion coefficient

$\kappa$ Permeability of porous medium

$\eta$ Dimensionless similarity variable 
$\psi$ Stream function

$\theta$ Dimensionless temperature

$\varphi$ Dimensionless concentration

$D_{B}$ Brownian diffusion coefficient

$D_{T}$ Thermophoresis diffusion coefficient

$M$ Magnetic parameter

$\mathrm{Gr}_{x}$ Local Grashof number

$\mathrm{Re}_{x}$ Local Reynolds number

$\mathrm{Nr}$ Buoyancy ratio

$K$ Permeability parameter

Pr Prandtl number

Le Lewis number

$\mathrm{Nb}$ Brownian motion parameter

Nt Thermophoresis parameter

$f$, $G$ Dimensionless stream functions

$(\rho \Phi)_{p}$ Effective heat capacity of a nanoparticle

$(\rho \Phi)_{f}$ Heat capacity of the fluid

$\tau_{\mathrm{wx}}$ Wall shear stresses in the $x$-direction

$\tau_{\mathrm{wz}}$ Wall shear stresses in the $z$-direction

$C_{f x}$ Skin friction coefficient in $x$-direction

$C_{f z}$ Skin friction coefficient in $z$-direction

$\mathrm{Nu}_{x}$ Local Nusselt number

$\mathrm{Sh}_{x}$ Local Sherwood number

$q_{w}$ Surface heat flux

$j_{m}$ Surface mass flux

Subscripts

$f$ Fluid

$p$ Nanoparticle

$w$ Condition at the surface

$\infty$ Ambient condition

Superscripts

' Differentiation w. r. t. $\eta$

Authors' contributions

All the authors have made substantive contributions to the article and assume full responsibility for its content. Both authors read and approved the final manuscript.

Funding

No fund for the conduction of this research.

Availability of data and materials

Data sharing is not applicable to this article as no datasets were generated or analyzed during the current study.

Competing interests

The authors declare that they have no competing interests.

Author details

${ }^{1}$ Department of Mathematics, Ambo University, Ambo, Ethiopia. ${ }^{2}$ Department of Mathematics, Wollega University, Nekemte, Ethiopia. 
Received: 29 July 2019 Accepted: 25 September 2019

Published online: 17 December 2019

\section{References}

1. Choi, S.U.S.: Enhancing thermal conductivity of fluids with nanoparticles. In: Proceedings of the ASME International Mechanical Engineering Congress and Exposition, vol. 66, pp. 99-105. ASME, FED 231/MD, San Francisco (1995)

2. Ittedi, S., Ramya, D., Joga, S.: MHD heat transfer of nanofluids over a stretching sheet with slip effects and chemical reaction. Int. J. of Lat. Eng. Res. Appl. 02, 10-20 (2017)

3. Sreekala, B., Janardhan, K., Ramya, D., Shravani, l.: MHD boundary layer nanofluid flow of heat transfer over a nonlinear stretching sheet presence of thermal radiation and partial slip with suction. Glob. J. of Pure Appl. Math. 13, 4927-4941 (2017)

4. Yohannes, K.Y., Shankar, B.: Heat and mass transfer in MHD flow of nanofluids through a porous media due to a stretching sheet with viscous dissipation and chemical reaction effects. Carib. J. Sci. Tech. 1, 1-17 (2013)

5. Ibrahim, W.: Magnetohydrodynamic (MHD) boundary layer stagnation point flow and heat transfer of a nanofluid past a stretching sheet with melting. Prop. Power Res. 6(3), 214-222 (2017) https://doi.org/10.1016/j.jppr.2017.07.002

6. Kasaeian, A., Azarian, R.D., Mahian, O., Kolsi, L., Chamkha, A.J., Wongwises, S., Pop, I.: Nanofluid flow and heat transfer in porous media: a review of the latest developments. Int. J. of Heat Mass Transf. 107, 778-791 (2017) https://doi.org/10. 1016/j.jijheatmasstransfer.2016.11.074

7. Ittedi, S., Ramya, D., Joga, S.: Partial slip effect of MHD boundary layer flow of nanofluids and radiative heat transfer over a permeable stretching sheet. Glob. J. of Pure Appl. Math. 13(7), 3083-3103 (2017)

8. Sulochana, C., Samrat, S.P., Sandeep, N.: Thermal radiation effect on MHD nanofluid flow over a stretching sheet. Int. J. of Eng. Res. in Africa. 23, 89-102 (2016). https://doi.org/10.4028/www.scientific.net/JERA.23.89

9. Haroun, N.A.H., Mondal, S., Sibanda, P.: Unsteady natural convective boundary-layer flow of MHD nanofluid over a stretching surface with chemical reaction using the spectral relaxation method: a revised model. Proc. Eng. 127, 18-24 (2015). https://doi.org/10.1016/j.proeng.2015.11.317

10. Reddy, C.H.A., Shankar, B.: Magnetohydrodynamics stagnation point flow of a nanofluid over an exponentially stretching sheet with an effect of chemical reaction, heat source and suction/injunction. World. J. Mech. 5, 211 - 221 (2015). https://doi.org/10.4236/wim.2015.511020

11. Li, Z., Ramzan, M., Shafee, A., Saleem, S., Al-Mdallal, Q.M., Chamkha, A.J.: Numerical approach for nanofluid transportation due to electric force in a porous enclosure. Micro. Tech. 25(6), 2501-2514 (2019)

12. Ramzan, M., Mohammad, M., Howari, F., Chung, J.D.: Entropy analysis of carbon nanotubes based nanofluid flow past a vertical cone with thermal radiation. Entropy. 21(7), 1-17 (2019). https://doi.org/10.3390/e21070642

13. Bilal, M., Ramzan, M.: Hall current effect on unsteady rotational flow of carbon nanotubes with dust particles and nonlinear thermal radiation in Darcy-Forchheimer porous media. J. of Therm. Analy. Calori. 1-11 (2019) https://doi.org/ 10.1007/s10973-019-08324-3

14. Lu, D., Ramzan, M., Mohammad, M., Howari, F., Chung, J.D.: A thin film flow of nanofluid comprising carbon nanotubes influenced by Cattaneo-Christov heat flux and entropy generation. Coatings. 9(5), 1-16 (2019). https://doi.org/10.3390/ coatings 9050296

15. Suleman, M., Ramzan, M., Ahmad, S., Lu, D., Muhammad, T., Chung, J.D.: A numerical simulation of silver-water nanofluid flow with impacts of Newtonian heating and homogeneous - heterogeneous reactions past a nonlinear stretched cylinder. Symm. 11(2), 1-13 (2019). https://doi.org/10.3390/sym11020295

16. Suleman, M., Ramzan, M., Ahmad, S., Lu, D.: Numerical simulation for homogeneous-heterogeneous reactions and Newtonian heating in the silver-water nanofluid flow past a nonlinear stretched cylinder. Phys. Scr. 94(8), 1-9 (2019) https://doi.org/10.1088/1402-4896/ab03a8

17. Madhu, M., Kishan, N., Chamkha, A.: Boundary layer flow and heat transfer of a non-Newtonian nanofluid over a nonlinearly stretching sheet. Int. J. of Numer. Meth. for Heat \& Fluid Flow. 26(7), 2198-2217 (2016)

18. Madhu, M., Kishan, N.: Finite element analysis of heat and mass transfer by MHD mixed convection stagnation-point flow of a non-Newtonian power-law nanofluid towards a stretching surface with radiation. J. of the Egyp. Math. Soci. 24(3), 458-470 (2016)

19. Macha, M., Naikoti, K., Chamkha, A.J.: MHD flow of a non-Newtonian nanofluid over a non-linearly stretching sheet in the presence of thermal radiation with heat source/sink. Eng. Comput. 33(5), 1610-1626 (2016) https:/doi.org/10.1108/ EC-06-2015-0174

20. Alebraheem, J., Ramzan, M.: Flow of nanofluid with Cattaneo- Christov heat flux model. Appl. Nano- sci. 1-11 (2019)

21. Faroog, U., Lu, D.C., Munir, S., Suleman, M., Ramzan, M.: Flow of rheological nanofluid over a static wedge. J. of Nanofluids. 8(6), 1362-1366 (2019)

22. Farooq, U., Lu, D., Munir, S., Ramzan, M., Suleman, M., Hussain, S.: MHD flow of Maxwell fluid with nanomaterials due to an exponentially stretching surface. Sci. reports. 9(1), 1-11 (2019) https://doi.org/10.1038/s41598-019-43549-0

23. Macha, M., Reddy, C.S., Kishan, N.: Magnetohydrodynamic flow and heat transfer to Sisko nanofluid over a wedge. Int. J. of Fluid Mech. Res. 44(1), (2017)

24. Aman, F., Ishak, A.: Mixed convection boundary layer flow towards a vertical plate with a convective surface boundary condition. Math. Prob. in Eng. 2012, 1-11 (2012)

25. Mahanthesh, B., Gireesha, B.J., Gorla, R.S.R.: Heat and mass transfer effects on the mixed convective flow of chemically reacting nanofluid past a moving/stationary vertical plate. Alex. Eng. J. 55, 569-581 (2016) https://doi.org/10.1016/j.aej. 2016.01.022

26. Ahamed, S.M., Mondal, S., Sibanda, P.: Unsteady mixed convection flow through a permeable stretching flat surface with partial slip effects through MHD nanofluid using spectral relaxation method. Open Phys. 15, 323-334 (2017)

27. Haroun, N.A.H., Sibanda, P., Mondal, S., Motsa, S.S.: On unsteady MHD mixed convection in a nanofluid due to a stretching/shrinking surface with suction/injection using the spectral relaxation method. Bound. Value Prob. 24, 1-17 (2015). https://doi.org/10.1186/s 13661-015-0289-5

28. Haroun, N.A.H., Mondal, S., Sibanda, P.: Effects of thermal radiation on mixed convection in a MHD nanofluid flow over a stretching sheet using a spectral relaxation method. Int. J. of Math. Comput. Phys. Elec. Comp. Eng. 11(2), 33-42 (2017) 
29. Khan, A.Q., Rasheed, A.: Mixed convection magnetohydrodynamics flow of a nanofluid with heat transfer: a numerical study. Math. Prob. in Eng. 2019, 1-14 (2019) https://doi.org/10.1155/2019/8129564

30. Reddy, C.R., Surender, O., Rao, C.V.: Effects of Soret, Hall and ion-slip on mixed convection in an electrically conducting Casson fluid in a vertical channel. Nonlinear Eng. 5(3), 167-175 (2016)

31. Reddy, C.R., Surender, O., Rao, C.V., Pradeepa, T.: Adomian decomposition method for Hall and ion-slip effects on mixed convection flow of a chemically reacting Newtonian fluid between parallel plates with heat generation/absorption. Prop. Power Res. 6(4), 296-306 (2017) https://doi.org/10.1016/j.jppr.2017.11.001

32. Srinivasacharya, D., Shafeeurrahaman, M.: Mixed convection flow of nanofluid in a vertical channel with hall and ion-slip effects. Front. in Heat Mass Transf. 8(11), 1-8 (2017). https://doi.org/10.5098/hmt.8.11

33. Srinivasacharya, D., Shafeeurrahaman, M.: Hall and ion slip effects on mixed convection flow of nanofluid between two concentric cylinders. J. of the Assoc. of Arab Univ. for Basic Appl. Sci. 24, 223-231 (2017) https://doi.org/10.1016/j. jaubasr.2017.03.002

34. Md. Shafeeurrahman, D. Srinivasacharya, Radiation effect on mixed convection flow of nanofluid between two concentric cylinders with Hall and lon-slip effects, AAM: Int. J. Special Issue No. 4 (2019) 82 - 96.

35. Su, X: Hall and ion-slip effects on the unsteady MHD mixed convection of $\overline{\mathrm{Cu}}$-water nanofluid over a vertical stretching plate with convective heat flux. Indian J. of Pure Appl. Phys. 55, 564-573 (2017)

36. Motsa, S.S., Shateyi, S.: The effects of chemical reaction, Hall, and ion-slip currents on MHD micropolar fluid flow with thermal diffusivity using a novel numerical technique. J. of Appl. Math. 2012, 1-30 (2012). https://doi.org/10.1155/2012/689015

37. Bilal, M., Hussain, S., Sagheer, M.: Boundary layer flow of magneto-micropolar nanofluid flow with Hall and ion-slip effects using variable thermal diffusivity. Bull. of the polish acad. of sci. tech. sci. 65(3), 383-390 (2017). https://doi.org/ 10.1515/bpasts-2017-0043

38. Ghara, N., Maji, S.L., Das, S., Jana, R., Ghosh, S.K.: Effects of Hall current and ion-slip on unsteady MHD Couette flow. Open J. of Fluid Dynam. 2, 1-13 (2012) https://doi.org/10.4236/ojfd.2012.21001

39. Ali, F.M., Nazar, R., Arifin, N.M., Pop, l.: Effect of Hall current on MHD mixed convection boundary layer flow over a stretched vertical flat plate. Meccanica. 46, 1103-1112 (2011). https://doi.org/10.1007/s11012-010-9371-3

40. Motsa, S.S., Makukula, Z.G.: On spectral relaxation method approach for steady von $\mathrm{Ka}^{\underline{E}} \mathrm{rma}{ }^{\underline{E}} \mathrm{n}$ flow of a Rreiner-Rivlin fluid with Joule heating, viscous dissipation and suction/injection. Cent. Europ. J. Phys. 11(3), 363-374 (2013). https://doi. org/10.2478/s11534-013-0182-8

41. Shateyi, S., Marewo, G.T.: A new numerical approach for the laminar boundary layer flow and heat transfer along a stretching cylinder embedded in a porous medium with variable thermal conductivity. J. of Appl. Math. 2013, 1-7 (2013) https://doi.org/10.1155/2013/576453

42. Shateyi, S., Marewo, G.T.: A new numerical approach of MHD flow with heat and mass transfer for the UCM fluid over a stretching surface in the presence of thermal radiation. Math. Prob. in Eng. 2013, 1-8 (2013) https://doi.org/10.1155/ 2013/670205

43. Ramzan, M., Bilal, M., Chung, J.D., Farooq, U.: Mixed convective flow of Maxwell nanofluid past a porous vertical stretched surface - an optimal solution. Results in Phys. 6, 1072-1079 (2016) https://doi.org/10.1016/j.rinp.2016.11.036

44. L. N. Trefethen, Spectral methods in MATLAB. vol. 10 of Software, Environments, and Tools, SIAM, Philadelphia, Pa, U.S.A. (2000).

45. Grubka, L.J., Bobba, K.M.: Heat transfer characteristics of a continuous stretching surface with variable temperature. ASME J. Heat Transf. 107, 248-250 (1985)

\section{Publisher's Note}

Springer Nature remains neutral with regard to jurisdictional claims in published maps and institutional affiliations.

\section{Submit your manuscript to a SpringerOpen ${ }^{\circ}$ journal and benefit from:}

- Convenient online submission

- Rigorous peer review

- Open access: articles freely available online

- High visibility within the field

- Retaining the copyright to your article

Submit your next manuscript at $\boldsymbol{s p r i n g e r o p e n . c o m ~}$ 